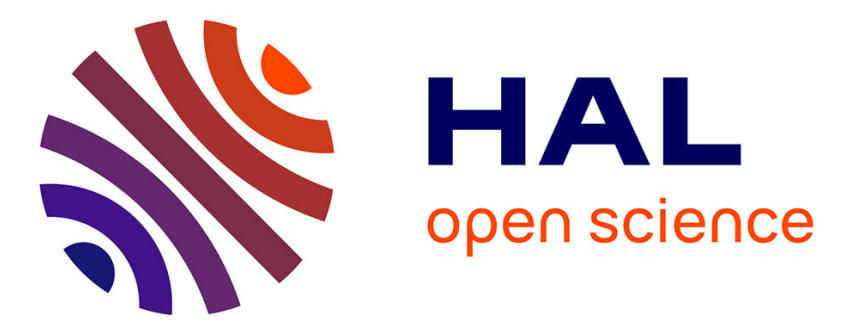

\title{
RECENT DEVELOPMENTS IN THE EXPERIMENTAL STUDIES OF XANES
}

\author{
J. Petiau, G. Calas, P. Sainctavit
}

\section{To cite this version:}

J. Petiau, G. Calas, P. Sainctavit. RECENT DEVELOPMENTS IN THE EXPERIMENTAL STUDIES OF XANES. Journal de Physique Colloques, 1987, 48 (C9), pp.C9-1085-C9-1096. 10.1051/jphyscol:19879197 . jpa-00227314

\section{HAL Id: jpa-00227314 https://hal.science/jpa-00227314}

Submitted on 1 Jan 1987

HAL is a multi-disciplinary open access archive for the deposit and dissemination of scientific research documents, whether they are published or not. The documents may come from teaching and research institutions in France or abroad, or from public or private research centers.
L'archive ouverte pluridisciplinaire HAL, est destinée au dépôt et à la diffusion de documents scientifiques de niveau recherche, publiés ou non, émanant des établissements d'enseignement et de recherche français ou étrangers, des laboratoires publics ou privés. 


\title{
RECENT DEVELOPMENTS IN THE EXPERIMENTAL STUDIES OF XANES
}

\author{
J. PETIAU, G. CALAs and P. SAINCtAVIT* \\ Laboratoire de Minéralogie - Cristallographie, Universités \\ Paris VI et VII (CNRS UA-09), 4, Place Jussieu, F-75252 Paris \\ Cedex 05, France \\ "Saint-Gobain Recherche, 39, Quai Lucien Lefranc, \\ F-93304 Aubervilliers Cedex, France
}

\begin{abstract}
Résumé - La spectroscopie d'absorption X au voisinage des seuils informe sur la structure électronique locale et sur l'arrangement géométrique autour de l'élément absorbant. Cet article présente des résultats expérimentaux récents en les classant d'après l'interprétation qui en est donnée.
\end{abstract}

\begin{abstract}
X-Ray absorption spectroscopy in the vicinity of absorption edges contain information about the electronic structure and about the geometrical arrangement around the absorbing atom. This paper presents recent experimental studies in relation to the type of interpretation which is given.
\end{abstract}

X-ray Absorption Near Edge Structure (XANES) currently designs the absorption structures corresponding to transitions from core levels to empty bound states or to continuum states within 30 $80 \mathrm{eV}$ above the vacuum level. The possibilities of XANES in giving information about the local electronic structure and about the geometrical arrangement of atoms around the absorbing atomic species have long been recognized but the quantitative interpretation was recently improved by new theoretical advances and new experimental facilities.

The only quantitative information extracted from the spectra has long been the "chemical shift" due to the more or less important contribution of the valence orbitals on the core state and interpreted in term of oxidation number. The recent period has shown that such an interpretation is over-simple even if the comparison of energy positions remains an element of information. The expected information is either of electronic nature or of geometrical nature, though both aspects are intimately related.

Experimentally, X-Ray Absorption Spectroscopy (XAS) is relatively easy to carry out with Synchrotron Radiation Sources. On the other hand XAS has a low energy resolution. The intrinsic limitation is given by the core hole width ( 0.1 to several $\mathrm{eV}$ according to the atomic number and the core level) [1 -2]. The apparent facility and the intrinsic resolution limitation should not mask the fact that the main recent progresses towards quantitative interpretation of the XANES energy range lay on experimental improvements and innovations. It is necessary to have good absolute measurements of the absorption coefficient; this requires harmonic free monochromatized beams. Careful energy calibrations with reference systems are needed since nor the Fermi level neither the vacuum level directly appear in the spectra. To get the whole information available the experimental energy width emerging from the monochromator has to be made significantly smaller than the core level width; it can generally be attained if appropriate Bragg reflections are chosen.

It is clear that the results given by XANES are complementary to those given by the other spectroscopies giving access to either full or empty electronic states. The more direct comparison is with BIS ( inverse photoemission) which also probes unoccupied states. There is no room here to spread out these complementarities though it appears in recent works that they are the key for understanding complex systems. 
A last general remark should be made. XANES probes the unoccupied electronic states having a selected angular momentum and relative to a selected atomic species.The main characteristics of absorption spectroscopy are these atomic and angular momentum selectivities. It makes it a valuable tool in structurally and electronically complex systems. For example it is able to give information about the $5 \mathrm{f}$ electronic states of an actinide element embedded at a $1 \%$ concentration in a glass containing half the Mendeleev table in its composition [3]. As a consequence XANES studies, as EXAFS studies, are undertaken in an extraordinary diversity of systems.

Experimental results cannot be separated from theoretical developments. We chose to present the results according to the type of theoretical interpretations which are associated.

The paper is therefore organised as follows :

- In section 1, analysis of the spectra in term of partial local density of electronic states are described. They are based upon band structure calculations and prove the predominance of one electron processes. This type of theoretical approach can evidently only be used in systems with long range order.

- Section 2 concerns interpretations made by molecular orbital considerations or related methods. This led to the more numerous publications. These methods are mainly used to establish correlations between the geometry at the atomic site of the absorbing element and the features energies. This type of approach only concems the very first part of XANES.

- In section 3, multiple scattering analysis are reported. They are analysed more extensively by Natoli in the present conference. Up to now calculations have been performed for a small number of compounds.

- The question of the importance of multiple scattering processes depending on the photoelectron energy is of primordial interest for the validity of EXAFS treatment. It is the object of section 4.

- The electronic transition is generally described in term of a one electron process. Final state effects, as electron-electron interactions and interactions of electrons with the core hole, play roles which are more or less important depending on the type of compound. These effects may be observed in highly correlated electronic systems by the splitting of the final state configurations. Some examples of these effects are given in section 5 .

- Section 6 is devoted to the first results in spin-dependent XANES.

\section{1 - INTERPRETATIONS IN TERM OF DENSITIES OF STATES}

The band structure approach gives a good description of the whole spectrum for $3 \mathrm{~d}$ and $4 \mathrm{~d}$ transition metals [4-5-6-7]. This work is not very recent but it remains a constant reference in XANES interpretation. The most important paper applies the method to palladium and gives an exhaustive list of earlier studies [7].

The main consequence is that the absorption coefficient can be factorized into an atomic term and a solid-state term. The atomic term has a smooth energy dependence in the XANES region, after a possible strong absorption peak at the edge. By contrast the solid-state term which represents the partial density of states at the site of the absorbing atom varies rapidly with energy and is characteristic of the band structure. In a large energy range but near the edge the density of states is mainly determined by the geometrical arrangement so that spectra of metallic elements having the same 
crystalline structure evidence strong similarities. It is for example the case with XANES structures of copper and palladium $\mathrm{K}$-edges in the cubic fcc structure. In this method the final states are described as eigenstates of the ground state potential (without core-hole) and in the muffin-tin approximation. In reference [7] Müller presents absorption spectra of palladium at various edges. He successfully compares each of them with the projected density of states corresponding to the dipole-allowed final state symmetries.

A systematic comparison between experimental and calculated K-edge spectra for $3 \mathrm{~d}$ transition elements and their oxides has been published [8]; it shows that the experimental structures are reproduced from the edge to $200 \mathrm{eV}$ after it. Another paper studied the L-edges of the metals $\mathrm{Rh}, \mathrm{Pd}$, $\mathrm{Ag}$, In and Sn [9]. All these works confirm that one electron processes are responsible for most of the features.

Apart from these studies concerning metals or simple compounds, comparisons between experimental spectra and partial densities of states computed in a band structure framework are seldom possible by lack of calculations concerning energies well above the Fermi level. Experimentalists try generally to interprete the very first part of the spectra. Few examples are now given of such recent studies.

Palladium L-edges spectra for palladium clusters embedded in a carbon matrix have been compared to the spectra of bulk palladium. A sizable shift to higher energies and a decrease in the absolute value of the absorption coefficient are observed at the Fermi level for small clusters. It is interpreted as a change of the density of states of $d$ character at the Fermi level [10-11].

The L II-III spectra of bulk silicon and of the Si (111) $2 \times 2$ surface have been interpreted by comparison with tight binding calculations of the s density of states; main features are in concordance except by an energy shift which is interpreted as due to the effect of the core hole on the s final state [12]. K-edge spectra of each element of the semiconductor $\mathrm{CuFeS}_{2}$ (chalcopyrite) have been compared with the three projected densities of states[13].

LII-III edges of transition elements present intense absorption peaks due to the dipole-allowed character of the $2 p \rightarrow d$ transition ("white-lines"). Changes in the intensity, position and shape of this peak could be an access to changes in the density of empty d-states. It has been used but difficulties appeared since the $\mathrm{L}_{\mathrm{WI}} / \mathrm{L}_{\mathrm{II}}$ white-line intensity ratio is often different from the statistical ratio, proving atomic effects added to density of states effects. It is especially true when the $\mathrm{d}$ band is nearly full and this deviation increases from the first- to the third-row transition elements as a consequence of spin-orbit effects. This has been carefully discussed in references [9-14-15]:

\section{2- MOLECULAR ORBITAL INTERPRETATIONS}

In opposition to band structure interpretations this local approach is convenient to answer structural questions in systems having no long range order.

In the simplest interpretations the features are interpreted in term of non bonding and antibonding states. The photoelectron is considered as having been excited into an empty bound state. In the electric-dipole approximation, a given orientational symmetry of the absorbing site leads to a selection 
of symmetry allowed transitions. Comparisons between calculated levels and the fine structure energies close to the edge evidences the actual geometry in the compound under study.

The experimental works supported by this type of interpretations are numerous in various domains such as solid state chemistry, coordination chemistry, biological chemistry ... It is impossible to report all papers, even with restriction to the recent ones, because they are published in a very large range of journals of various specialities. Only some typical examples are given and arbitrarily chosen.

The parallel between band structure and molecular orbital descriptions is clearly shown in a paper concerning $\mathrm{PdAl}_{3}$ and $\mathrm{PdCl}_{2}$ [16]. The comparison is made between L-edges in these insulating solids and in the metal palladium. The white-line maximum reflects the lowest unoccupied molecular orbital having partial d-character. The significance of the white-line shift between different compounds is discussed in term of molecular orbital energies. A thorough study of vanadium compounds has shown the correlation of various features near the edge with the vanadium oxidation and coordination states [17].

The most easily interpretable structures are the "pre-edges" which are present in the K-edge spectra of transition elements and characterize empty "d-like" states. All transitions from s-states to "d-like" states are forbidden by dipolar selection rules for absorbing atoms which are inversion symmetry centers. The features of low intensities in systeme having local octahedral symmetry result from a partial p-character of the electronic density at the site of the absorbing atom. The far more intense pre-edges for tetrahedral symmetry have become an usual way to recognize 4-fold tetrahedral sites from 6-fold octahedral sites in unknown systems. For example a systematic study of titanium K-edges in minerals has been published [18]. The increasing percentage of 6-fold sites as crystalline nucleation progresses in silicate glasses where titanium plays a nucleating role has been determined [19]. Titanium and vanadium K-edges are also the subject of reference [20] which concerns ( $\mathrm{Ti}, \mathrm{V}) \mathrm{O}_{2}$ rutile solid solutions. In reference [17] the strength of the pre-edge transition is found to be mainly dependent on the size of the "molecular cage" formed by the vanadium atom and the ligand atoms. For those transition elements $(\mathrm{Ti}, \mathrm{V}, \mathrm{Fe}$ ) which can be encountered in various oxidation states and with various coordination numbers, a careful study of pre-edge positions, intensities and structures can answer many questions. This type of studies rest fundamentally on comparisons with known compounds; crystal field, ligand field and molecular orbital considerations give a theoretical support to these comparisons.

Some features remain non interpreted in these schemes. The most typical failure of one electron ground-state interpretations is given by the titanium $\mathrm{K}$-edge for 6 -fold titanium atoms. A three peaks pre-edge feature is allways observed. Only two of them are interpreted; a multielectron process is generally invoked to explain the small peak at the lowest energy [20].

In transition metal compounds experiments are generally performed at the cation edges. A study of oxygen $\mathrm{K}$-edges in a variety of crystalline oxides have been published [21]. As expected an intense white-line is observed; its position is correlated with the oxygen coordination.

Coordination chemistry provides the experimentalist with compounds where cation sites have various definite local arrangements. They are models for the determination of local symmetry and chemical state 
deduced from edge features. For example the characterization of edge features corresponding to various coordination symmetries has been made in iron porphyrins [22], in vanadyl porphyrins [23], in a series of Mn compounds [24] etc ... The thermally induced spin crossover in iron and cobalt complexes has also been investigated [25].

Compounds of technological interest can now be investigated by this atomic selective method. For example the degree of charge localization in organic charge-transfer salt (HMTSF-TCNQ, HMTSF-TFTCNQ) have been related to edge features at selenium K-edge [26]. A recent work has been devoted to the inclusion of copper clusters within a conducting polymer (poly-3-methylthiophene) [27]. A time resolved in situ investigation of th process has been achieved by dispersive XAS. The $\mathrm{K}$-edges of $\mathrm{Cu}^{1+}, \mathrm{Cu}^{2+}$ and $\mathrm{Cu}^{0}$ (metal) are non-ambiguously assignable. The evolution from $\mathrm{Cu}^{2+}$ ions in solution to an intermadiate $\mathrm{Cu}^{1+}$ state and finally to $\mathrm{Cu}^{0}$ in the metallic clusters is observed in a series of spectra recorded during $3.6 \mathrm{~s}$ every $7.2 \mathrm{~s}$.

XANES has become for ten years one of the spectroscopy extensively used in the study of f-compounds. In this domain models negiecting the effect of the core-hole potential and multielectronic process can seldom be used. This is briefly presented in section 5 . The present paper only gives some references in this topic.

The edge structure is currently used in the study of mixed-valent rare-eatrh compounds. Two well separated contributions corresponding to each $4 \mathrm{f}$ configuration are observed in the $\mathrm{L}_{\mathrm{III}}$ absorption edges; the intensities give the percentage of each configuration [28-29-30-31-32-33-34-35]. The method is often criticized because of the possible existence of multielectronic processes; this question is carefully discussed in a review paper by Krill [36]. Moreover edge features contain information about the degree of localization of unoccupied electronic states. M-edges directly reflect the f-electron states. It is of a special interest for actinide compounds where 5f-electrons are in an intermediate situation between localized and delocalized states [3-37-38-39-40]. In cases where $4 \mathrm{f}$ localization is strong, dipole-selected multiplets are observed and interpreted [41-42].

A very important possibility is the use of the orientational dependence of the absorption spectra for anisotropic systems. This has become possible with linearly polarized synchrotron radiation beams. In the dipole approximation the matrix element for the transition is given by $\left\langle\phi_{f}|\mathbf{E} . r| \phi_{i}\right\rangle$. Varying the orientation of $\mathbf{E}$ with respect to the surface or crystallographic directions changes the absorption coefficient [43], Templeton and Templeton have published the first papers in polarized XAS [44-45-46]. Polarization dependence is expected in all non-cubic single crystals. Polarized measurements of a series of crystalline copper complexes are used to discriminate the final-state symmetry of various transitions [47]. In a former paper the same measurements put in evidence the electric quadrupole character of a dipole-forbiden transition in the pre-edge region of copper compounds [48].

For chemisorbed molecules on metallic surfaces the polarized XANES depends on the geometrical arrangement of the molecule on the substrate. Numerous papers are published on this topic [49-50-51-52-53-54]. For example the orientation of small organic molecules $\left(\mathrm{CO}, \mathrm{HCOOH}, \mathrm{CH}_{3} \mathrm{OH}\right)$ 
on $\mathrm{Cu}(100)$ has been determined by the polarization dependant intensities of the $\sigma^{*}$ and $\pi^{*}$ orbital transitions. This was possible because edge structure is dominated by intramolecular scattering resonances. Another of group of papers is devoted to lamellar compounds. Several studies concern graphite and intercalated graphite compounds [55-56-57-58]. The absorption peaks in graphite correspond to $\sigma^{*}$ orbitals (in the basal plane) and $\pi^{*}$ orbitals (perpendicular to the basal plane) are clearly resolved. When the polarization vector lies within the basal plane, $\sigma^{*}$ final states are selected while when $E$ has a large perpendicular components, states of $\pi^{*}$ symmetry are selected [55].

\section{3 - MULTIPLE SCATTERING INTERPRETATIONS}

The single scattering approach has proved to be correct for high energy photoelectrons. The electron scattering is generally weak above $50-100 \mathrm{eV}$ except in the forward and backward directions so that multiple scattering processes are very unlikely and the single scartering theory suffices. It is no longer true for electrons-of low kinetic energy and a multiple scattering description becomes necessary.

First multiple scattering calculations by multiple scattered wave SCF X-alpha calculations in small clusters are reported in papers which remain references several years after their publication [59-60-61-62-63-64-65]. They are full multiple scattering calculations taking the scattering process as a whole. These calculations are improved year after year in various directions : more realistic exchange correlation potentials (energy dependent Dirac-Hara potential [66], real part of Hedin-Lunqvist potentials), use of non real potentials taking into account the limited. mean free path of the photoelectron (imaginary part of Hedin-Lundqvist potentials [67-68-69] ), use of non-muffin tin potentials [70] etc.... Most of the calculations are performed with non self-consistent potentials. The calculated spectra are in good agreement with the measurements in numerous systems. The size of the cluster and the geometrical arrangement are the only physical parameters that can be varied.

Recent calculations in zinc blende for the K-edge spectra of zinc and of sulfur show the necessity of growing the cluster up to 8 shells for S (99atoms) and to 6 shells for $\mathrm{Zn}$ (71atoms) in order to obtain the first experimental features $(0-10 \mathrm{eV})$ with correct relative amplitudes [71]. The calculation for successive addition of sulfur and zinc shells evidences clearly that the scattering cross section of zinc is low. This agrees well with the calculated phase shifts for low energy photoelectrons [72].

The main interest for the experimentalist is to understand the structural information contained in the XANES spectrum. Calculations are manageable in various symmetry conditions as long as the cluster contains only few atoms. A series of studies have concemed compounds of known structures to check the validity of the method.

The first calculations in solids have concerned metallic $\mathrm{Ag}, \mathrm{Cu}$ and $\mathrm{Mn}$ [61-62]. They were applied later to other 3d transition metals [73], to diamond [74] and to III-V semiconductors [75-76]. For the two fcc metals $\mathrm{Cu}$ and $\mathrm{Ag}$, the main features are already reproduced with a three shell cluster (43 atoms) as well as for a four shell cluster (55atoms). For Mn which has complicated structures where the environment of manganese atoms is highly distorted the calculations for hypothetical fcc or bcc 
structures do not reproduce well the experimental spectra of alpha-Mn. On the other hand the experimental amplitudes of oscillations are smaller in manganese than in well ordered structures. It is previsible that the disorder in the environment "washes out" the structures and by the way the remaining features are due to multiple scattering processes in the very first shells. This method could be well suited to investigate small geometrical changes in highly disordered systems. .

The importance of multiple scattering effects in crystalline and amorphous silicon has also been recently investigated. This question will be discussed below as the support of section 4 [77].

These results seem to confirm the fact that multiple scattering processes in the XANES range have significant probability within a volume of the same order as single scattering processes in the EXAFS energy range. The actual mean free path of the photoelectron is limited at low kinetic energies by the effect of the core hole life time and at higher energies by inelastic processes [78]. The result of this compensation is that the actual volume of access of the photoelectron remains in the same range all along the XANES and EXAFS regions.

At the present time the purpose has been the calculation for given absorbing and scattering atoms in various symmetries and the comparison with experimental spectra. However the full multiple scattering calculations do not give idea about the respective importance of successive orders. The nth order contribution is the cross secton resulting of all the scattering events involving $\mathbf{n}$ atoms (including the absorbing atom). When full multiple scattering calculations are performed, it is not impossible that single scattering processes are the only processes of some importance down to the edge.

EXAFS taking into account only the single scattering processes cannot give more than the pair correlation functions, as do the diffraction methods lying in the Born approximation. On the contrary multiple scattering interpretations can in principle give access to higher order correlation functions, in particular bond angles. This can however only be attained if the contribution of each scattering order in the overall spectrum is separately calculated [67]. Natoli has shown the validity of a linear development of the absorption coefficient $\alpha(\mathrm{k})$ in function of the scattering processes of successive orders:

$$
\alpha(k)=\alpha_{0}(k)\left[1+\Sigma \kappa_{n}(k)\right]
$$

$\alpha_{0}(k)$ is the atomic absorption coefficient.

The application of this method for tetrahedral and octahedral symmetries has been made for manganese oxides in liquid solutions [67-68-69]. It has shown that the energy range where third and fourth orders should be considered is wide. As expected the full multiple scattering is necessary near the edge.

Further geometrical investigation needs to separate the contributions of different paths to a given scattering order. Scattering in the incoming direction is far more important than for large angles. It has been inferred for this reason that only unidirectional scatterings (labelled "1st -type" MS) are predominant in compact structures [79]. This effect gives the "focussing effect" or "shadowing effect" in the EXAFS interpretation of fcc structures where the fourth shell is exactly in the same direction as the first one viewed from the central atom. The increased contribution of the fourth shell to the EXAFS spectrum has long been observed in copper. It is actually a multiple scattering process. 
In surface studies the importance of the polarization dependence has been emphasized in the second section. These studies have received a new attention in the multiple scattering framework where the contribution of each polarization can be separately calculated [80-81]. The possible geometrical arrangements of oxygen atoms on nickel or copper substrates have been checked and compared to experiments [82-83]. The very low symmetry of adsorbed oxygen makes these calculations time-consuming. A new fast working code has been developed to test all the possible geometrical arrangements. In the latest updated code the multiple-scattering equations are solved first within each shell by a perturbation expansion, and then between the shells themselves and the central atom. This method leads to the determination of the exact oxygen symmetry and to the determination of the bond distance between the oxygen atom and the substrate surface. As in the order expansion method developed before, this method allows to compare the relative importance of different intrashell scattering events [84]. In these cases of single atoms on surfaces it should be pointed out that a multiple scattering study is necessary to understand the geometry while for chemisorbed molecules the intamolecular scattering is predominent and allows a direct understanding of the geometry [80].

A useful pragmatical analysis may be made from a "scale law" concerning the energy position of multiple scattering resonances of the photoelectron in the continuum. It works reasonably well for the comparison of systems where all things (geometrical arrangement, electronic situation) are nearly identical except the variable distance between the absorbing atom and its neighbours [85-86]. It has been systematically investigated and verified for tetrahedral environments of various $3 \mathrm{~d}$ transition elements coordinated to oxygen atoms [87] or to sulfur atoms [13]. This effect has been evidenced for uranium and thorium environments in oxides of identical structures $\left(\mathrm{ThO}_{2}\right.$ and $\mathrm{UO}_{2}$ having the fluorine structure); the "distance-energy" formula $\left(E-E_{0}\right) d^{2}=c s t$ has therefore been used to measure $\mathrm{U}-\mathrm{O}$ and Th-O distances in glasses from the known distances in crystals [3]. The values are in agreement with those measured by EXAFS. It is of an easy use in these cases because XANES structures are mainly due to the first neighbours.

\section{4 - SINGLE VERSUS MULTIPLE SCATTERING}

The first interest is to know whether it is possible to analyse the near-edge part of the spectrum by the EXAFS formalism. It is very important in the soft X-ray range where the spectrum is often very short and for light scattering atoms which have important backscattering amplitudes only for photoelectrons of low energies.

The second interest is to know whether the higher-order contributions are of enough importance compared to the single-scattering one to give an access to higher order correlation functions.

As for the first point it has been suggested that the discrepancy between plane-wave EXAFS calculations and experiments in the low-energy domain of XANES is due mainly to the break down of the plane-wave approximation [87-88-89-90-91-92-93]. In that hypothesis spectra can be analysed in term of single-scattering all the way to the absorption edge if an appropriate curved-wave calculation is 
used. There is no room here for a valuable presentation of the current state of the question. Effort is made to give to experimentalists ab initio calculations with the curved-wave formalism in the form of distance-dependent backscattering amplitudes and phase shifts calculated close to the absorption edges [91] or as analytical expressions [90-92-93]. The improvement of structural results obtained by replacing usual plane-waves by curved-waves has been checked in several model systems $(\mathrm{Cu}$, $\mathrm{NiO} . .$.$) .$

However that does not prove that multiple scattering processes are unimportant in the XANES range. It has been said in the preceding section that the "focussing" effect ("type 1" MS) is important in the EXAFS domain. Whether it is the only important contribution has been controversial.

In $\mathrm{NiO}$ the importance of "type 2 " effects is concentrated in the first $20 \mathrm{eV}$ above the edge [94]. In all the calculations relative to $\mathrm{O}$ on $\mathrm{Ni}$ and $\mathrm{Cu}$ substrates, the multiple-scattering spectra were compared to the single-scattering ones for the same structure [83]. For a given symmetry the multiple scattering and single scattering spectra are very similar except in the first $15 \mathrm{eV}$ after the edge. Single scattering calculations are not much sensitive to slightly different symmetries, and produce a common average spectrum rather different from the experimental one in the region of the first $15 \mathrm{eV}$. On the contrary the multiple scattering calculations are more symmetry dependent in this region and a good agreement can be found with the experimental spectrum for one of the tentative symmetries. Therefore this near edge region is able to discriminate between various local arrangements and then the multiple scattering method is the only one able to give non-ambiguous structural answers in these compounds.

In silicon, the multiple scattering contribution has been studied by substraction of the calculated curved-wave spectrum from the experimental $\kappa(k)[77]$. The comparison of the obtained difference spectrum with the contributions of the various possible paths for multiple scattering shows the predominance of a triangular path (third order term in the development) which uses an atom of the first shell and an atom in the second shell. The other types of triangular paths are far less important. At the same time Fourier transforms of $\mathrm{Si}$ and $\mathrm{SiC}$ spectra from the edge gives contributions up to the seventh atomic shell at right distances if phase shifts are supposed independent of the energy of the photoelectron for low kinetic energies [95].

\section{5 - FINAL-STATE AND MULTIELECTRONIC EFFECTS}

A fundamental question for the experimentalist is to know if XAS actually brings information about the system in its ground state. The photoabsorption final state is certainly not the ground state because of the existence of the core hole. However in most cases the ground state information can be deduced from a modelization of the final state. The two simplest choices for the final state are either to take it as identical to the ground state of the absorbing atom in the compound, with its $Z$ electrons, or to take it as identical to the ground state of the " $\mathrm{Z}+1$ " atom. The validity of either model is not evident; it could depend on the photoelectron energy which governs the sudden versus adiabatic approximation and on the metallic versus insulating character of the material. The difference between the final state and the "Z" or " $Z+1 "$ ground state should be taken explicitly into account in many cases.

In the pre-edges interpretations for example the relative positions of the fine structures are not generally 
"atomic" the orbitals are, the more attracted by the core hole they get. The energies and splitting values of fine structures in XAS spectra are then not in general equal to the ground state values or to the values measured in low-energy spectroscopies. In band calculations the final state wave functions are always eigen states of the ground state [7]. In the multiple scattering approach the final state potential is either a " $Z$ " or a more or less screened " $Z+1$ " potential [60]. It has been shown that one electron models using final or ground states are only correct in the two limits of transitions to nearly empty and nearly filled bands respectively [96]. These one electron interpretations are not appropriate in many systems.

In highly correlated systems as rare earth compounds multielectron configurations are responsible for multiplet structures in XAS. In $\mathrm{NiO}, \mathrm{CeO}_{2}$ and other rare earth compounds, spectra cannot be interpreted unless interactions between the core hole, the atomic electrons and the photoelectron are taken into account [97-98-99-100-36]. Theoretical models have been extensively developed during the last years. The present experimental paper cannot review them. In few cases edge features have been interpreted as multielectron satellites : "shake-down" in $\mathrm{CuCl}_{2}$ [101], "shake-up" in $\mathrm{MnCl}_{2}, \mathrm{FeCl}_{2}$, $\mathrm{CoCl}_{2}[102]$ and in $\left[\mathrm{MnO}_{4}\right]^{-}$and $\left[\mathrm{CrO}_{4}\right]^{--}$in solutions [68].

\section{6 - SPIN-DEPENDENT X-RAY ABSORPTION}

A new and very promising domain is the investigation of the spin-density distribution in the unoccupied bands of magnetic materials [103-104-105-106].

The photoabsorption cross section of core levels contains a spin-dependent contribution if the final states are spin-polarized as in magnetic materials. Elliptically polarized incident photons give indeed partially polarized photoelectrons with the result of a near-edge absorption different for right and left circular polarizations. The spin-dependent term in the cross-section is proportional to the polarization transferred to the photoelectron $\left(10^{-2}\right.$ for the K-edge of iron) and to the difference in spin-density of the final states with their spin parallel and antiparallel to the spins of the magnetic electrons. It is therefore possible to determine the difference between the spin densities of either direction as a function of the energy above the Fermi level.

In the extended absorption region, the spin-dependence also exists and is expected to give information about the magnetic structure in the neighborhood of the absorbing atom; this is not the object of this paper.

Circularly polarized beams can be obtained from Synchrotron Radiation Sources either by choosing the appropriate part of the photon beam given by a bending magnet above and below the plane of the electron orbit or by using specially designed asymmetric wigglers.

First evidences of the effect concern the $\mathrm{L}_{\mathrm{III}}$ edge of gadolinium in $\mathrm{Gd}_{18} \mathrm{Fe}_{82}$ [103], a terbium iron garnet [105] and the $\mathrm{K}$ edge of iron [106]. In iron the measurements performed imply that the empty p-like band is populated preferentially by photoelectrons with spins in the direction of the $d$ electrons in the range of $2 \mathrm{eV}$ above the Fermi level. It proves the existence of a majority-spin band at that energy whereas the effect is inverse around $7 \mathrm{eV}$ and reveals a broader minority-spin band.

This method presents the advantages and the limitations of X-ray absorption spectroscopy. The 
atomic selectivity allows the investigation of spin densities localized on magnetic atoms embedded in complex materialsl or present as impurities. Another advantage is that it probes bulk states contrary to inverse photoemission. Limitations are the poor energy resolution and the low level of the spin-dependent part of the absorption coefficient $\left(10^{-4}-10^{-3}\right)$.

\section{References.}

1 - V.O.Kostroun, M.H.Chen, B.Crasemann. Phys. Rev. A $\underline{3}, 533$ (1971)

2 - W.Bambynek, B.Crasemann, R.W.Fink, H.U.Freund, H.Mark, C.D.Swift, R.E.Price, P.Venugopala Rao. Rev. Mod. Phys 44,716 (1972)

3 - J.Petiau, G.Calas, D.Petit-Maire, A.Bianconi, M.Benfatto and A.Marcelli; Phys.Rev.B 34, 7350 (1986)

4 - J.E.Müller, O.Jepsen, O.K.Andersen and J.W.Wilkins. Phys. Rev. Lett. 40.720 (1978)

5 - J.E.Müller, O.Jepsen and J.W.Wilkins. Solid State Comm.42, 365 (1982)

6 - G.Materlik, J.E.Müller and J.W.Wilkins, Phys. Rev. Lett. 50, 267 (1983)

7 - J.E.Müller and J.W.Wilkins. Phys. Rev. B 29, 4331 (1984)

8 - L.A.Grunes. Phys. Rev. B 27, 2111 (1983)

9 - T.K.Sham. Phys Rev. B 31 , 1888 (1985)

10 - M. De Crescenzi, M.Diociaiuti, P.Picozzi and S.Santucci. Phys.Rev.B 344334 (1986)

11 - M. De Crescenzi, E.Colavita, U.Del Pennino, P.Sassaroli, S.Valeri, C.Rinaldi, L.Sorba and S.Nannarone. Phys. Rev. B $\underline{32}, 612$ (1985)

12 - A.Bianconi, R.Del Sole, A.Selloni, P.Chiaradia, M.Fanfoni and I.Davoli (to be published)

13 - Ph. Sainctavit, G.Calas, J.Petiau, R.Kamatak, J.M.Esteva, G.E.Brown Jr. J. Phys.(Paris) 47. C8, 419 (1986)

14 - W.G.Waddington, P.Rez, I.P.Grant, C.J.Humphreys. Phys. Rev. B34, 1467 (1986)

15 - J.Zaanen, G.A.Sawatzky, J.Fink, W.Speier, J.C.Fuggle. Phys.Rev.B 32, 4905 (1985)

16 - T.K.Sham. Phys Rev. B 31 , 1903 (1985)

17 - J.Wong,F.W.Lytle, R.P.Messmer and D.H.Maylotte. Phys. Rev.B 30, 5596 (1984)

18 - G.A.Waychunas Amer. Miner. 72,89 (1987)

19. - A. Ramos, J.Petiau, G.Calas (to be published)

20 - B.Poumellec, J.F.Marucco and B.Touzelin Phys. Rev. B 35. 2284 (1987)

21 - G.E.Brown Jr., G.A.Waiychunas, J.Stöhr and F.Sette. J. Phys. (Paris) 47, C8, 685 (1986)

22 - M.Verdaguer, C.Cartier, M.Momenteau, E.Dartyge, A.Fontaine, G.Tourillion, A.Michalowicz. J. Phys. (Paris) 47, C8, 649 (1986)

23 - M.F.Ruiz-Lopez, D.Rinaldi, C.Esselin, J.Goulon, J-L.Poncet, R.Guilard. J.Phys. (Paris). 47, C8 637 (1986)

24 - C.Cartier, M.Verdaguer, S.Menage, J.-J.Girerd, J.-P.Tuchagues, B.Mabad. J. Phys. (Paris) 47, C8, 623 (1986)

25 - C.Cartier, P.Thuery, M.Verdaguer, J.Zarembowitch, A.Michailowicz. J. Phys. (Paris) 47, C8, 563 (1986)

26 - P.M.Grant, W.D.Gill, H.Morawitz, K.Bechgaard, D.E.Sayers. Phys. Rev. B $\underline{30}$, 6973 (1984)

27 - G.Tourillon, E.Dartyge, A.Fontaine and A.Jucha. Phys. Rev. Letters \$7, 603 (1986)

28 - A.Bianconi, A.Marcelli, M.Tomellini and I.Davoli. J. of Mag. Mag. Mat. 47-48, 209 (1985)

29 - W.D.Brewer, G.Kalkowski, G.Kaindl, F?Holtzberg. Phys. Rev, B32, 3676 (1985)

30 - D.Malterre, G.Krill, I.Durand, G.Marchal, M.F.Ravet. Phys. Rev. B34, 2176 (1986)

31 - E.Beaurepaire, J.P.Kappler, G.Krill. Solis State Comm. 57, 145 (1986)

32 - W.Niemann, W.Malzfeldt, P.Rabe, M.Lübcke. J. Phys. (Paris) 47, C8, 931 (1986)

33 - G.Wortmann, B.Perscheid, W.Krone. J. Phys. (Paris) 47, C8, 979 (1986)

34 - D.Malterre, A.Siari, J.Durand, G.Krill and G.Marshall. J. Phys. (Paris) 47. C8, 991 (1986)

35 - A.Bianconi, A.Marceili, H.Dexpert, R.Karnatak, A.Kotani, T.Jo and J.Petiau. Phys.Rev.B 35, 806 (1987)

36 - G.Krill. J. Phys. (Paris $47, \subseteq 8,907$ (1986)

37 - J.M.Lawrence, M.L. den Boer, R.D.Parks and J.L.Smith. Phys. Rev. B 29. 568 (1984)

38 - G.Kalkowski, G.Kaindl, W.D.Brewer, W.Brone. Phys. Rev. B 35, 2667 (1987)

39 - R.C.Karnatak, J-M. Esteva, H.Dexpert, M.Gasgnier, P.E.Caro and L.Albert. Phys. Rev. B, 36, 1745 (1987)

40 - H.Dexpert, R.C.Karnatak, J-M.Esteva, J.P.Connerade, M.Gasgnier, P.E.Caro and L.Albert. Phys. Rev. B, 36.1736 (1987)

41 - B.T.Thole, D.R.Cowan, G.A.Sawatzky, J.Fink, J.G.Fuggle. Phys.Rev.B 31, 6856 (1985)

42 - B.T.Thole, G.van der Laan, J.C.Fuggle, G.A.Sawatzky, R.C.Karnatak, J.M.Esteva. Phys.Rev.B 325107 (1985)

43 - D.D.Vvedensky, D.K.Saldin, J.B.Pendry. Surface Science 162, 909 (1985)

44 - D.H.Templeton and L.K.Templeton. Acta Cryst. A36, 237 (1980)

45 - D.H.Templeton and L.K.Templeton. Acta Cryst. A38, 62 (1982)

46 - D.H.Templeton and L.K.Templeton. Acta Cryst. A4L 133 (1985)

47 - J.E.Penner-Hahn, T.A.Smith, B.Hedman, K.O.Hodgson and S.Doniach. J.Phys. (Paris). 47, C8 1197 (1986)

48 - J.E.Hahn, R.A.Scott, K.O.Hodgson, S.Doniach, S.Desjardins, E.I.Solomon. Chem.Phys.Lett. 88, 595 (1982)

49 - J.Stöhr, J.L.Gland, W.Eberhardt, D.Outka, R.J.Madix, F.Sette, R.J.Koestner, U.Doebler Phys.Rev.Lett. $\underline{51}, 2414(1983)$

50 - F.Sette, J.Stöhr, E.B.Kollin, D.J.Dwyer, J.L.Gland, J.L.Robbins, A.L.Johnson. Phys.Rev.Lett. 54,935 (1985) 
51 - A.Bianconi, A.Congiu-Castellano, P.J.Durham, S.S.Hasnain, S.Phillips. Nature (Letters) $\underline{318} 685$ (1985)

52 - M.Bader, J.Haase, K.-H.Frank, A.Puschmann and A.Otto. Phys.Rev.Letters 56, 1921 (1986)

53 - M.Bader, A.Puschmann and J.Haase. Phys.Rev.B (Rapid Comm.) 33, 7336 (1986)

54 - G.Tourillon, A.Fontaine, R.Garrett, M.Sagurton, P.Xu and G.P.Williams. Phys.Rev.B 35, 9863 (1987)

55 - R.A.Rozenberg, P.J.Love, V.Rehn. Phys.Rev. B 33, 4034 (1986)

56 - J.L.Feldman, W.T.Elam, A.C.Ehrlich, E.F.Skelton, D.D.Dominguez, D.D.L.Chung, F.W.Lytle. Phys.Rev.B 33. 7961 (1986)

57 - C.Fretigny, D.Bonnin and R.Cortes. J.Phys. (Paris), 47, C8 869 (1986)

58 - G.Loupias, J.Chomilier, J.Tarbes, I.Ascone, J.Goulon, D.Guerard, N.Elalem. J.Phys.(Paris) 47, C8 891 (1986)

59 - F.W.Kutzler, C.R.Natoli, D.K.Misemer, S.Doniach and K.O.Hodgson. J.Chem.Phys. 73(7). 3274 (1980)

60 - C.R.Natoli, D.K.Misemer, S.Doniach and F.W.Kutzler. Phys.Rev.A 22,1104 (1980)

61 - P.J.Durham, J.B.Pendry and C.H.Hodges. Solid State Comm. 38,159 (1980)

62 - G.N.Greaves, P.J.Durham, G.Diakun and P.Quinn. Nature 294. 139 (1981)

63 - F.W.Kutzler, K.O.Hodgson and S.Doniach. Phys. Rev. A 26, 3020 (1982)

64 - J.B.Pendry. Comments Solid State Phys. 10(6), 219 (1983)

65 - J.B.Pendry. J.Phys.(Paris) 46, C9, 93 (1985)

66 - S.H.Chou, F.W.Kutzler, D.E.Ellis, G.K.Shenoy, T.I.Morrison, P.A.Montano. Phys. Rev. B 31, 1069 (1985)

67 - M. Benfatto, C.R.Natoli, A.Bianconi, J.Garcia, A.Marcelli, M.Fanfoni, I.Davoli. Phys.Rev.B 34, 5774 (1986)

68 - J.Garcia, M.Benfatto, C.R.Natoli, A.Bianconi, I.Davoli and A.Marcelii. Solid State Comm. $\underline{58}, 595$ (1986)

69 - M.Benfatto, C.R.Natoli, J.Garcia and A.Bianconi. J.Phys.(Paris) $47 \underset{\mathrm{C} 8}{\underline{8}}, 25$ (1986)

70 - C.R.Natoli, M.Benfatto and S.Doniach. Phys. Rev. A, 34, 4682 (1986)

71 - Ph.Sainctavit, J.Petiau, G.Calas, M.Benfatto and C.R.Natoli. (This volume)

72 - C.R.Natoli and M.Benfatto. I.Phys. (Paris) 47.C8, 11 (1986)

73 - M.Kitamura, S.Muramatsu and C.Sugiura Phys. Rev:B 33, 5294 (1986)

74 - M.Kitamura, C.Sugiura and S.Muramatsu. Solid State Comm. 62, 663 (1987)

75 - T.Matsuura, T.Fujikawa and H.Oyanagi. J. Phys. Soc. Japan. 58,2837 (1984)

76 - H.Oizumi, J.lizuka, H.Oyanagi, T.Fujikawa, T.Ohta and S.Usami. Jpn. J.Appl. Phys.24, 1475 (1985)

77 - A.Bianconi, A.DiCicco, N.V.Pavel, M.Benfatto, A.Marcelli, C.R.Natoli, P.Pianetta and J.Woicik (to be published)

78 - A. Di Cicco, A.Bianconi and N.V.Pavel. Solid State Comm. 61, 635 (1987)

79 - G.Bunker and E.A.Stern. Phys. Rev. Lett. 52,1990 (1984)

80 - D.Norman, J.Stöhr, R.Jaeger, P.J.Durham and J.B.Pendry. Phys. Rev, Letters 51, 2052 (1983)

81 - F.Antonangeli, M.piacentini, R.Girlanda, G.Martino, E.S.Giulano. Phys.Rev.B 32, 6644 (1985)

82 - D.D.Vvedensky, D.K.Saldin, J.B.Pendry. Surface Science 162, 845 (1985)

83 - D.D. Vvedensky, J.B.Pendry, U.Döbler and K.Baberschke. Phys.Rev. B, 35, 7756 (1987)

84 - D.D.Vvedensky and J.B.Pendry, Surface Science 162, 903 (1985)

85 - A.Bianconi, M.Dell'Ariccia, A.Gargano and C.R.Natoli. "EXAFS and Near-Edge Structure" Springer Series on Chem.Phys. 27, 43 (1983)

86 - C.R.Natoli. "EXAFS and Near-Edge Structure III" Springer Proceedings in Physics 2.38 (1985)

87 - A.Bianconi, E.Fritsch, G.Calas and J.Petiau. Phys. Rev. B, 32, 4292 (1985)

88 - J.E.Müller and W.L.Schaich. Phys. Rev. B, 27, 6489 (1983)

89 - W.L.Schaich. Phys. Rev.B, 22, 6513 (1984)

90 - J.J.Rehr, R.C.Albers, C.R.Natoli and E..Stem. Phys Rev B $\underline{34}, 4350$ (1986)

91 - A.G.McKale, G.S.Knapp and S.K.Chan. Phys. Rev. B 33, 841 (1986)

92 - S.J.Gurman, N.Binsted and L.Ross. J. Phys. C, 17, 143 (1984)

93 - S.J.Gurman, N.Binsted and I.Ross. J. Phys. C, 19,1845 (1986)

94 - D.D. Vvedinsky and J.B.Pendry. Phys.Rev.Lett. (Comment) 54, 2725 (1985)

95 - P.Lagarde and A.M.Flank. J.Phys.(Paris) 47, 1389 (1986)

96 - E.A.Stem and J.J.Rehr. Phys. Rev. B 27, 3351 (1983)

97 - I.Davoli, A.Marcelli, A.Bianconi, M.Tomellini, M.Fanfoni. Phys.Rev.B 33, 2979 (1986)

98 - G.van der Laan, J.Zaanen, G.A.Sawatzky, R.Kamatak, J.M.Esteva. Phys.Rev.B 33, 4253 (1986)

99 - A.Kotani and T.Jo. J. Phys. (Paris) 47, C8, 915 (1986)

100 - E.Beaurepaire, F. Le Normand, G.Krill. J. Phys. (Paris) 47, C8, 961 (1986)

101 - R.A.Bair and W.A.Goddart III. Phys. Rev. B 22, 2767 (1980)

102 - E.A.Stem. Phys. Rev. Letters. 49, 1353 (1982)

103 - E.Keller and E.A.Stem. "EXAFS and Near-Edge Structure III" Springer Proceedings in Physics 2. 507 (1985)

104 - B.T.Thole, G.van der Laan and G.A.Sawatzky. Phys. Rev. Letters. 55 , 2086 (1985)

105 - G. Van der Laan, B.T.Thole, G.A. Sawatzky, J.B.Goedkoop, J.G.Fuggle; J-M. Esteva, R.Karnatak, J.P.Remeika and H.A. Dabwoska. Phys. Rev. B, 34, 6529 (1986)

106 - G.Schiit, W.Wagner, W.Wilhelm, P.Kienle, R.Zeller, R.Frahm and G.Materlik. Phys.Rev.Lett. 58,737 (1987) 